\title{
EL CONCEPTO DE EXPOSICIÓN SIMBÓLICA EN KANT
}

\section{THE CONCEPT OF SYMBOLIC EXPOSITION IN KANT}

\author{
ÀLEX MUMBRÚ MORA \\ Universidad de Barcelona
}

\begin{abstract}
RESUMEN: En el dominio teórico, la validez objetiva de nuestros conceptos depende de su posible referencia a la intuición, ya sea por medio de la mostración de un ejemplo del mismo o de su construcción en la intuición a priori. La exigencia de una referencia intuitiva se extiende también a aquellas representaciones que escapan por principio a toda posible sensibilización, si bien sin pretensión cognoscitiva alguna. Este sería el caso de los conceptos de razón en general, de los que hace uso el discurso moral en cuanto esencialmente heterogéneo de nuestros conceptos teóricos. En el presente artículo se analiza la naturaleza, funcionamiento y lugar sistemático del concepto de «exposición simbólica», en cuanto modo alternativo de referencia al mundo sensible por el que se nos hacen patentes aspectos de una representación que carece de todo referente intuitivo directo, i.e. las ideas o conceptos de razón. En este sentido, se muestra el conjunto de relaciones por las que el ámbito estético deviene el campo de sensibilización del dominio moral.

Palabras Clave: lenguaje, significado, exposición simbólica, estética, moralidad.

ABSTRACT: The objective validity of our conceptual representations in the theoretical domain depends on their possible reference to intuition, either through presenting an example or constructing an a priori intuition. Although it has no cognitive purpose, the demand of an intuitional reference also applies to those representations which are essentially not capable of being sensitized. This would be the case of rational concepts in general, which are used by moral speech. The main aim of our paper is to analyze the nature, function and systematic place of symbolic exposition, as an alternative way of referring to the sensible world that allows us to present some aspects of the representations, i.e. the ideas or rational concepts, which lack any direct intuitive reference. More specifically, we will analyze the set of relations by which aesthetics becomes the field where the moral domain is rendered sensible.
\end{abstract}

KEYWORDS: language, meaning, symbolic exposition, aesthetics, morality. 


\section{Introducción}

Aun cuando las reflexiones sobre el lenguaje por parte de Kant son escasas, resulta razonable plantear la existencia de una concepción semántica implícita en el conjunto de la filosofía trascendental ${ }^{1}$. La cuestión del significado se corresponde en Kant a la pregunta por el fundamento de la relación entre una representación universal dada y el particular que le corresponde. En este sentido habla Kant de «sensibilización» (Versinnlichung), «exposición» (Darstellung) o «hipotiposis» (Hypotypose) como aquel proceso por el que nos es en general posible proporcionar un contenido intuitivo a una determinada representación conceptual ${ }^{2}$.

En el dominio teórico, el fundamento de la relación particular-tipo reside en el esquema en cuanto modo general de proceder por el que la imaginación es capaz de esbozar una imagen que sea adecuada a un concepto dado (empírico o sensible puro) ${ }^{3}$. Ahora bien, como consecuencia del carácter no sensible que es propio de la dimensión moral del ser racional finito, cabe preguntarse por el

\footnotetext{
${ }^{1}$ Con respecto al papel que juega el lenguaje en la filosofía de Kant pueden consultarse: MARKIS, D., «Das Problem der Sprache bei Kant», en B. Scheery G. Wohlfart (ed.), Dimensionen der Sprache in der Philosophie des Deutschen Idealimus, Würzburg, Königshausen und Neumann, 1982, pp. 110-154; Villers, J., Kant und das Problem der Sprache, Konstanz, Verlag am Hockgraben, 1997.

${ }^{2} \mathrm{Cfr}$. Ku, $\$ 59$ (Ak, V, 351). Como es habitual, la citación de los textos kantianos sigue la edición de la Academia (Ak), consignando el volumen en números romanos y la página en numeración arábiga, a excepción de la Crítica de la razón pura ( $\mathrm{KrV})$ que se cita de acuerdo con el procedimiento usual, según la paginación de las ediciones A o B. Las traducciones de los textos de Kant son del autor de este artículo.

${ }^{3}$ Los principales estudios en esta línea de investigación son: BuTTS, R. E., «Kant’s Schemata as Semantical Rules», en L. W. Beck (ed.), Kant's Studies Today, La Salle, Open Court, 1969, pp. 290300; Thompson, M., «Singular Terms and Intuitions in Kant's Philosophy», Review of Metaphysics, Diciembre de 1972, pp. 314-43; PIPPIN, R. B., «The Schematism and Empirical Concepts», KantStudien, 67 (1976), pp. 156-171; Nolan, J. P., «Kant on Meaning: Two Studies», Kant-Studien, 70 (1979), pp. 113-130; HanNA, R., «Kant's Theory of Empirical Judgment and Modern Semantics», History of Pbilosophy Quarterly, vol. 7, n. 3 (Julio de 1990), pp. 335-351; del mismo autor, «The trouble with Truth in Kant's Theory of Meaning», History of Philosophy Quarterly, vol. 10, n. 1 (Enero de 1993), pp. 1-20; LOHMAR, D., «Kants Schemata als Anwendungsbedingungen von Kategorien auf Anschauungen», Zeitschrift für philosophische Forschung, 45 (1991), pp. 77-92; del mismo autor, «Husserl's Type and Kant's Schemata. Systematic Reasons for Their Correlation or Identity», en D. Welton (ed.), The New Husserl, Indiana University Press, 2003, p. 93-124; Düssing, K., «Schema und Einbildungskraft in Kants Kritik der reinen Vernunft», en Günter Gawlick y Lothar Kreimendahl (ed.), Aufklärung und Skepsis. Studien zur Pbilosophie und Geistesgeschichte des 17. und 18. Jahrbunderts, Stuttgart, Frommann-Holzboog, 1995, pp. 47-71.
} 
tipo de referencia a la intuición que puede aceptarse para nuestros conceptos morales, sin que haya quebranto alguno del presupuesto fundamental acerca de la heterogeneidad entre el mundo sensible y el inteligible.

Dado que el ámbito de objetos sobre el que se aplican es uno y el mismo tanto para el dominio teórico como para el práctico, i.e. «el conjunto (Inbegriff) de los objetos de toda experiencia posible en cuanto se les considera como meros fenómenos» ${ }^{4}$, la sensibilización del ámbito de la moralidad acontece mediante un modo alternativo de referencia al conjunto de los fenómenos que integran el mundo sensible. En esta trama discursiva se inscribe el concepto de «exposición simbólica» (symbolische Darstellung) ${ }^{5}$ en cuanto referencia analógica a un objeto de la intuición cuyo objetivo consiste en hacer aprehensible aspectos que son propios de una representación (las ideas o conceptos de razón) que escapa por definición a toda exposición directa en la sensibilidad ${ }^{6}$.

Como veremos, en el ámbito estético se produce una exposición simbólica del campo de la moralidad, esto es, encontramos elementos sensibles que ilustran analógicamente aspectos que son característicos del dominio práctico ${ }^{7}$. El objetivo del presente trabajo es dilucidar la plétora de relaciones que, por medio de la noción de «símbolo», se establecen entre los ámbitos estético y moral.

\footnotetext{
${ }^{4} \mathrm{KU}$, Einleitung, II (Ak, V, 174).

${ }^{5} \mathrm{KU}, \int 59$ (Ak, V, 351). Si bien la primera aparición del término «exposición» (Darstellung) con el sentido de «presentación sensible» la encontramos en el segundo Prólogo a KrV (BXXXIIXXXIII), es en la tercera crítica y en La religión dentro de los límites de la mera razón [Religion] donde aparece con mayor frecuencia; cfr. FELICITAS MunZEL, G., 〈»'The Beautiful is the Symbol of the Morally-Good». Kant's Philosophical Basis of Proof for the Idea of the Morally-Good», Journal of the History of Philosophy 33 (1995), p. 307.

${ }^{6}$ «Para exponer la realidad de nuestros conceptos se exigen siempre intuiciones. Si son conceptos empíricos, a estas últimas se las denomina ejemplos. Si son conceptos puros del entendimiento, se les llama esquemas. Si, por el contrario, se exige que sea expuesta la realidad objetiva de los conceptos de razón, i.e. de las ideas, y esto en vistas del conocimiento teórico de las mismas, se solicita algo imposible, ya que a las ideas de ninguna manera puede proporcionárseles ninguna intuición que les sea adecuada»; ibíd.

${ }^{7}$ La reflexión sobre la naturaleza y funcionamiento de la exposición simbólica, en cuanto presentación intuitiva de representaciones pertenecientes al ámbito de lo inteligible por medio de un procedimiento analógico, se inscribe en el marco de las constantes reflexiones kantianas encaminadas a establecer una clara diferenciación entre el conocimiento matemático y el filosófico, dado que si bien es cierto que ambas disciplinas utilizan el símbolo y la analogía, lo hacen en sentidos diferentes; cfr. KrV, B222/A179.
} 


\section{El concepto de exposición simbólica}

El examen relativo al estatuto y funcionamiento de todo discurso sobre el ámbito suprasensible, en el que cabe inscribir el moral en tanto que esencialmente heterogéneo con respecto al dominio teórico, es una preocupación constante en la reflexión kantiana. No en vano, la resolución de la antinomia necesidad-libertad (y, podríamos agregar, la investigación sobre la naturaleza y el modo de articulación del discurso moral desde la especificidad que le es propia) constituye una de las espuelas que dan inicio a la filosofía crítica $^{8}$. Ahora bien, no es hasta el apartado 59 de la Crítica de la facultad de juzgar (KU) que quedan terminológica y conceptualmente fijadas las dos formas en que nos es posible pensar toda posible relación particular-tipo (hipotiposis), sea la representación-tipo un concepto de razón o del entendimiento.

Así pues, con respecto al ámbito de lo suprasensible la constitución de nuestras facultades parece exigir cierto correlato intuitivo que nos haga «aprehensibles» $(f a l i c b)$ aquellas representaciones que rehúyen por principio toda sensibilización directa, sin presuponer no obstante que tal exposición detente valor cognoscitivo alguno. Este sería el caso de todos aquellos conceptos que pertenecen al ámbito de la moralidad, para los que Kant introduce la noción de «exposición simbólica», que tiene lugar «cuando a un concepto que sólo la razón puede pensar y al que ninguna intuición sensible puede serle adecuada, se le subsume una intuición con respecto a la cual el proceder de la facultad de juzgar coincide de un modo meramente analógico con aquello que observa cuando esquematiza, i.e. en la regla de tal proceder solamente, mas no en la intuición misma; coincide por tanto según la forma de la reflexión meramente, y no en lo que respecta al contenido»?

En cuanto relación de «hipotiposis», toda exposición simbólica presenta las siguientes características:

\footnotetext{
${ }^{8}$ Cfr. Carta a Ch. Garve, 21. September 1798 (Ak, XII, 257).

${ }^{9} \mathrm{Ku}, \$ 59(\mathrm{Ak}, \mathrm{V}, 351)$. Para una historia del concepto de «símbolo», desde su introducción como término con impronta filosófica en el Banquete de Platón, pasando por Plotino y su empleo por parte de Schiller y Hegel; cfr. JANKE, W., «Das Symbol», Philosophisches Jahrbuch 76 (1968/69), pp. 164-180; GuYER, P., Kant and the Experience of Freedom, New York, Cambridge University Press, 1993, pp. 39ss.
} 
a) el «tipo» es una idea o concepto de razón, i.e. una representación tal que no hay intuición sensible (empírica o pura) alguna que le sea plenamente adecuada, esto es, que pueda subsumírsele directamente (mediante la mostración de un ejemplo o construyendo a priori el concepto en la intuición) ${ }^{10}$;

b) la exposición simbólica es un modo intuitivo (que no discursivo) de representación; es decir, por exposición simbólica no entiende Kant la utilización de «signos sensibles» (sinnliche Zeichnen), i.e. meros «caracterismos» (Charakterismen) que no contienen nada que corresponda a la intuición del objeto y que, como es el caso de las palabras y los símbolos algebraicos, se emplean para designar conceptos ${ }^{11}$;

c) la exposición simbólica es un modo intuitivo indirecto de representación; que no es posible encontrar intuición alguna que sea plenamente adecuada a un concepto de razón significa que no puede mostrarse un ejemplo del mismo (concepto empírico) ni construirse a priori en la intuición (concepto sensible puro). Ahora bien, dado que el conjunto de los objetos del mundo sensible constituye el campo de aplicación tanto del dominio teórico como del práctico, el referente intuitivo en la exposición simbólica de un concepto de razón debe expresar de manera analógica aspectos de una representación que rehúye por principio toda posible sensibilización, es decir, mediante el reconocimiento de una igualdad en la relación entre dos pares de elementos heterogéneos entre $s 1^{12}$.

Así, por ejemplo, el funcionamiento mecánico de un molino de viento y todas las características que podemos obtener de su concepto (automatismo, unidi-

${ }^{10}$ «Cuando a un concepto puede serle dada la correspondiente intuición a priori, se dice que este concepto es construido. Si se trata solamente de una intuición empírica, se la denomina un mero ejemplo del concepto. La acción de añadir la intuición al concepto se llama en ambos casos exposición (exhibitio) del objeto, sin la que (puede tener lugar indirecta o directamente) no puede haber conocimiento alguno»; Fortschritte (Ak, XX, 325).

${ }^{11}$ Cfr. Ku, $\int 59$ (Ak, V, 351ff). El concepto de «sensibilización» (Versinnlichung) debe entenderse en términos de un «hacer-intuitivo» (Veranschaulichung) por el que proporcionamos una determinada imagen (ámbito intuitivo) a nuestros pensamientos (ámbito discursivo); cfr. FLACH, W., «Zu Kants Lehre von der symbolischen Darstellung», Kant-Studien 73 (1982), p. 455.

12 «En filosofía, las analogías significan algo muy diferente a aquello que representan en las matemáticas. En éstas son fórmulas que expresan la igualdad de dos relaciones entre magnitudes, y son siempre constitutivas, de tal modo que si son dados tres miembros de la proporción, también es dado el cuarto, i.e. puede ser construido. Ahora bien, en filosofía la analogía no es la igualdad de dos relaciones cuantitativas sino cualitativas, donde a partir de los tres miembros dados tan sólo puedo conocer y dar a priori la relación con un cuarto, y no este cuarto miembro mismo, si bien tengo una regla para buscarlo en la experiencia y un rasgo para descubrirlo en ella»; KrV, B222/A179. 
reccionalidad, rigidez, monotonía, etc.) exhiben intuitivamente la articulación de un estado despótico por medio de la equivalencia en la relación que encontramos en sus respectivos elementos (molino-aspas/déspota-súbditos) ${ }^{13}$. Cabe encontrar multitud de tales exposiciones simbólicas en nuestro lenguaje ordinario y, en particular, en la mayor parte de términos metafísicos (v. gr. la definición lockeana del concepto de substancia como «portador» de los accidentes) ${ }^{14}$;

d) en la exposición simbólica de un concepto de razón, la facultad de juzgar ejecuta pues una doble tarea: determina el objeto de acuerdo con el concepto que le corresponde (facultad de juzgar determinante), refiriendo a su vez ese objeto a una representación con la que no tiene una relación directa y cuyo objeto no es intuitivamente dable, pero al que expresa analógicamente. Es decir: en la exposición simbólica de una idea o concepto de razón, la referencia a la intuición se da por analogía con el procedimiento por el que la facultad de juzgar aprehende unitariamente una diversidad dada (la forma de la reflexión), i.e. la facultad de juzgar opera bajo la única restricción de hallar una representación intuitiva que exprese analógicamente aquello que escapa por definición a toda sensibilización cognoscitivamente válida ${ }^{15}$.

\section{La exposición simbólica del concepto de la libertad}

El sistema de los principios a priori del entendimiento establece las condiciones bajo las que caen necesariamente el conjunto de los fenómenos de la naturaleza en tanto que objetos de una experiencia posible. En particular, el principio de causalidad determina el modo en que tiene lugar toda posible relación entre los objetos que pertenecen al mundo sensible. En cuanto capacidad para determinarse a obrar con independencia de los estímulos procedentes de los sentidos, la idea de la libertad se define por su «carácter no-investigable» (Unerforschlichkeit) desde los parámetros del mundo natural ${ }^{17}$; y es por este motivo que Kant describe el campo de la moralidad en términos de su «mera oposición» (blo-

\footnotetext{
${ }^{13}$ Cfr. Ku, $\int 59$ (Ak, V, 352).

${ }^{14}$ Ibíd.

${ }^{15}$ Cfr. Ku, $\int 59$ (Ak, V, 352ff).

${ }^{16} \mathrm{Cfr} . \mathrm{KrV}, \mathrm{B} 163$.

${ }^{17}$ Cfr. Ku, Allg. Anmerk. zur Expox. Ästh. reflek. Urteilsk. (Ak, V, 275).
} 
be Entgegensetzung) con respecto al dominio de los conceptos teóricos ${ }^{18}$. Mas, a pesar de la esencial irreductibilidad que media entre ambos dominios, tanto en la experiencia de la belleza como en la de lo sublime se nos proporciona cierto correlato sensible de la idea de libertad, en cuanto idea sobre la que se fundamenta el campo de lo suprasensible ${ }^{19}$.

\subsection{El objeto bello}

Ante la belleza de un objeto, i.e. la imposibilidad de hallar una representación bajo la que subsumir la forma de aquello que se nos presenta en la intuición, apercibimos la «conformidad a fin sin fin» (Zweckmä igkeit obne Zweck) de nuestras facultades representativas, esto es, el armónico funcionamiento de entendimiento e imaginación en cuanto facultades involucradas de manera necesaria en la producción de una representación unitaria de carácter general ${ }^{20}$.

El espontáneo juego entre el entendimiento y la imaginación, que en la experiencia de lo bello opera sin someterse a la regla de ningún concepto en particular, es análogo a la ausencia de determinación externa que es propia del concepto de libertad en sentido práctico, i.e. la capacidad para determinarse a obrar prescindiendo de estímulos externos. Ahora bien, de la misma manera que la libertad no implica carencia absoluta de regla sino la «conformidad a ley» de nuestro arbitrio en la toma de decisiones (en particular, el sometimiento a aquella ley que el sujeto racional se da a sí mismo), la imaginación se atiene al marco legal establecido por los principios trascendentales del entendimiento tanto en el enjuiciamiento como en la producción de la belleza ${ }^{21}$. De este modo, en la experiencia de la belle-

\footnotetext{
${ }^{18}$ Cfr. Ku, Einleitung, I (Ak, V, 171).

${ }^{19} \mathrm{Cfr}$. Ku, Einleitung, II (Ak, V, 174).

${ }^{20}$ Cfr. I Einleitung Ku (Ak, XX, 233).

${ }^{21}$ Cfr. JANKE, W., op. cit., p. 175. En este mismo sentido, cabe afirmar que el objeto bello expone simbólicamente el concepto de la buena voluntad (guter Wille). Que «la buena voluntad es buena en sí misma, es decir, ni por aquello que efectúa o produce, ni por su idoneidad en la consecución de un fin propuesto, sino tan sólo mediante el querens [Grundlegung (Ak, IV, 394)], significa que la bondad de la buena voluntad reside en la mera forma del querer con independencia de cualquier fin exterior a la misma, de tal modo que puede afirmarse que en ella se da también una «conformidad a fin sin fin» y, por ende, que el objeto bello simboliza la naturaleza de la buena voluntad y la causalidad que le es propia. Sobre este punto, cfr. COHEN, T., "Why Beauty is a Symbol of Morality?», en 'T. Cohen (ed.), Essays in Kant's Aesthetics, Chicago, The University of Chicago Press, 1982, pp. 232ss.
} 
za se da una exposición simbólica de la idea de la libertad, esto es, se nos proporciona un referente intuitivo que patentiza analógicamente una representación que escapa por definición a toda sensibilización directa ${ }^{22}$. O, expresado en los ya célebres términos de Schiller: la belleza es la libertad en el fenómeno ${ }^{23}$.

\subsection{Las ideas estéticas}

En la medida en que el sujeto es incapaz de proporcionar concepto alguno que le sea plenamente adecuado, en la obra de arte bella las facultades cognoscitivas del ser racional finito se demoran (sich verweilen) en la contemplación de una representación que se caracteriza por mantener constantemente en vilo nuestra atención, conservando de manera activa a la imaginación y el entendimiento en su libre juego ${ }^{24}$. Cabe concebir pues la belleza en general (ya sea natural o artística) como el ámbito en el que se intentan exponer aquellas representaciones de la imaginación que «dan mucho que pensar» (viel zu denken veranlaben), i.e. las «ideas estéticas» (ästhetische Idee) ${ }^{25}$. Precisamente porque no descubrimos concepto alguno que les sea del todo adecuado, y porque nos mueven constantemente a ir más allá de los límites de nuestra experiencia, Kant las denomina $\left\langle\right.$ ideas» ${ }^{26}$.

En la medida en que su riqueza y potencial significativo excede todo concepto determinado, nuestras facultades representativas se sitúan allende lo dado en la sensibilidad, siendo así que la imaginación del ser racional finito apercibe su libertad con respecto a las leyes de asociación a que se somete en su uso empírico, y opera ateniéndose de manera exclusiva a la regla de su proceder reflexivo en general $^{27}$. De este modo, por medio de las ideas estéticas se da asimismo

${ }^{22}$ Cfr. Felicitas Munzel, G., op. cit., p. 309; Paton, H. J., The Categorical Imperative, Hutchinson \& Ltd., 1947, cap. XIV, App. \2; GUYER, P., op. cit., p. 316 y p. 99 ss.

${ }^{23} \mathrm{Al}$ respecto, puede consultarse Guyer, P., op. cit., cap. 3; Martínez MarzoA, F., De Kant a Hölderlin, Madrid, Visor, 1992, pp. 35-41.

${ }^{24} \mathrm{Cfr}$. Ku, $\$ 12$ (Ak, V, 222).

${ }^{25} \mathrm{Cfr}$. Ku, $\$ 49$ (Ak, V, 313ff).

${ }^{26}$ Ibíd. Para una reflexión sobre el concepto de «idea estética» y su lugar sistemático en la reflexión estética kantiana, cfr. LÜTHE, R., «Kants Lehre von den ästhetischen Ideen», Kant-Studien 75 (1984), pp. 65-74.

${ }^{27}$ «La imaginación (en tanto que facultad cognoscitiva productiva) es ciertamente muy poderosa en la creación (Schaffung) de, por así decir, otra naturaleza a partir del material que le proporciona 
una exposición indirecta de la idea de la libertad, puesto que esta capacidad de la imaginación para ubicarse ultra aquello que proporcionan los sentidos es análoga a la facultad que tiene el ser racional finito para determinarse a obrar autónomamente.

\subsection{El objeto sublime}

Por medio del objeto sublime se exhibe también indirectamente el concepto de la libertad. El sentimiento de lo sublime patentiza la inadecuación que media entre el conjunto de nuestras facultades representativas y aquello que se nos presenta en la intuición, ya sea a causa del carácter irresistible de su fuerza (sublime dinámico), o de la incapacidad de nuestra imaginación en su esfuerzo por sensibilizar la idea de totalidad exigida por la razón (sublime matemático).

Es justamente en la conciencia de la disposición «contraria a fin» (zweckwidrig) de la representación de un objeto con respecto a nuestras facultades que el ser racional finito apercibe no obstante la mutua conformidad que existe entre la imaginación, en su capacidad de proseguir indefinidamente la aprehensión de aquello que se le presenta en la intuición, y la razón en cuanto facultad que exige la absoluta totalidad en la serie de las condiciones con respecto a cualquier condición dada.

El objeto sublime constituye pues «aquello que, tan sólo poder pensarlo, da prueba de una facultad de nuestro espíritu que sobrepasa toda medida de los sentidos» ${ }^{28}$. Y, puesto que este índice de independencia con respecto a aquello que proviene de los sentidos es análogo a la capacidad que tiene el ser racional finito para determinarse de acuerdo con los principios que él mismo se proporciona, la representación de un objeto sublime expone de manera indirecta el concepto de la libertad.

la efectiva. Nos entretenemos con ella donde la experiencia se nos presenta demasiado cotidiana; la transformamos, si bien siempre según leyes analógicas, pero también según principios que residen más arriba en la razón (...); con lo que sentimos nuestra libertad con respecto a la ley de asociación (de que depende el uso empírico de aquella facultad), de acuerdo con la cual nosotros tomamos prestado material de la naturaleza, pudiendo ser transformado por nosotros en algo totalmente diferente, algo que supera la naturaleza»; $\mathrm{Ku}, \$ 49$ (Ak, V, 314).

${ }^{28} \mathrm{Ku}, \S 25$ (Ak, V, 250). 


\section{4, La experiencia de lo sublime y el sentimiento moral}

Cabe establecer asimismo una clara analogía entre el «sentimiento moral» y el efecto que la representación de un objeto sublime ocasiona en nuestras facultades. La ley moral se erige en el debido fundamento de determinación de nuestro arbitrio, siendo condición necesaria si bien no suficiente para poder aseverar el valor moral pleno de una acción, dado que ésta puede haber sido ejecutada conforme al deber sin que ello implique que el motivo último resida en la ley misma $^{29}$. Así, una de las cuestiones que cabe plantearse es de qué modo acercamos las exigencias de incondicionalidad propias de la ley moral al dominio de los motivos impulsores de la acción, e investigar cuál es el efecto que la ley moral produce en el conjunto de nuestras facultades representativas.

Dado el carácter sensiblemente condicionado — que no determinado- del arbitrio del ser racional finito, las exigencias de incondicionalidad de la ley moral se nos presentan como una imposición, de ahí que el concepto de deber moral tome en nosotros la forma de un imperativo categórico. Es decir: la ley moral tiene primeramente un efecto negativo (displicente) sobre nuestras facultades; nos humilla puesto que ningunea nuestra pretensión de satisfacer los estímulos que proceden de los sentidos.

Por otro lado, en la medida en que revela una dimensión en nosotros que va más allá de nuestro ser natural y que es capaz de imponerse a todo condicionamiento sensible, la ley moral nos eleva por encima de la «animalidad» que nos es propia y deviene objeto de «respeto» (Achtung $)^{30}$. Y nos ensalza ciertamente porque descubrimos que esta ley proviene de nosotros mismos, de tal modo que la conciencia de nuestra capacidad para sobreponernos a todo obstáculo en nuestro actuar acaba revirtiendo positivamente en la promoción misma de esta actividad. A la comunión de ambos momentos — humillación y elevación (respeto por nuestra condición moral) — la denomina Kant «sentimiento moral», del que cabe afirmar que es el único sentimiento cuya relación con nuestras facultades puede conocerse a priori ${ }^{31}$.

\footnotetext{
${ }^{29} \mathrm{Cfr} . \mathrm{KpV}(\mathrm{Ak}, \mathrm{V}, 72)$.

${ }^{30} \mathrm{Cfr} . \mathrm{KpV}$ (Ak, V, 74).

${ }^{31}$ Cfr. $\mathrm{KpV}(\mathrm{Ak}, \mathrm{V}, 73)$.
} 
En este contexto, es de fundamental importancia subrayar el hecho de que el sentimiento moral no constituye el criterio de moralidad de nuestras acciones, sino el efecto que las exigencias de incondicionalidad de la razón ejercen sobre la sensibilidad del ser racional finito ${ }^{32}$. De no ser así, correríamos el riesgo de que la ética kantiana deviniera un mero sentimentalismo moral, por el que el criterio de corrección radicaría en que nos sintiéramos humillados o elevados en el establecimiento de un determinado objeto como fin de nuestra acción. El sentimiento de respeto, por el contrario, es el modo en que la ley moral comparece como resorte de nuestro arbitrio en la toma de decisiones ${ }^{33}$.

Es posible establecer pues una relación de analogía entre el sentimiento de lo sublime y el efecto que la ley moral produce en el ser racional finito, ya que en ambos se da un recíproco movimiento descendente-ascendente de nuestras facultades ${ }^{34}$ :

a) por un lado, el quebranto de nuestras pretensiones sensibles mediante un elemento cuya fuerza se nos impone incondicionalmente, produciendo un sentimiento de «humillación» (Demütigung) hacia nuestra persona;

b) por otro, la conciencia de la existencia de una dimensión en nosotros que no es reducible a nuestro ser natural y que ocasiona un sentimiento de «respeto» hacia la misma ${ }^{35}$. En este sentido afirma Kant que el sentimiento de lo

\footnotetext{
${ }^{32}$ Cfr. Grundlegung (Ak, IV, 401Anm).

33 «Y, de este modo, el respeto por la ley no es el móvil para la moralidad, sino que es la moralidad misma considerada subjetivamente como móvil»; $\mathrm{KpV}(\mathrm{Ak}, \mathrm{V}, 76)$.

${ }^{34}$ La relación entre el sentimiento moral y la experiencia de lo sublime se constata también en la «Oda al deber» presentada por Kant en KpV, y que parece ser el contrapunto a la «Oda a la alegría» de Schiller:

«iDeber! Tú que portas tan gran y sublime nombre, nada preciado tomas en ti que conlleve zalamería sino que exiges sometimiento... ¿Dónde se encuentra la raíz de tu noble origen, que rechaza orgulloso todo parentesco con las afecciones, una raíz de la que procede la condición inexcusable de aquel valor que únicamente los seres humanos pueden darse a sí mismos?»; $\mathrm{KpV}$ (Ak, V, 86). La analogía de este fragmento con Schiller la debemos a GRONDIN, J., «Zur Phänomenologie des moralischen 'Gesetzes'. Das kontemplative Motiv der Erhebung in Kants praktischer Metaphysik», Kant-Studien 91 (2000), p. 390.

35 «El sentimiento de la inadecuación de nuestra capacidad para alcanzar una idea que es ley para nosotros es respeto»; KU, \27 (Ak, V, 257).
} 
sublime en la naturaleza es, en última instancia, un sentimiento de respeto hacia nuestra propia condición moral, a la humanidad (Menschbeit) en nosotros.

Aún más: la conciencia de la excelencia de la razón con respecto a toda medida/condicionamiento sensible se traduce en la atracción que la representación sublime produce en nuestra imaginación que se ve forzada a perseverar en su pretensión de alcanzar lo suprasensible ${ }^{36}$. Este empecinamiento puede considerarse asimismo un elemento análogo a la virtud entendida como la capacidad para hacer aquello que se considera correcto, sobreponiéndose a todo obstáculo o impedimento externo ${ }^{37}$. En este sentido afirma Kant que el concepto de deber es presentado intuitivamente de un modo más adecuado por el sentimiento de lo sublime, precisamente porque el modo en que se hace patente es mediante el continuo rechazo de nuestros intereses sensibles ${ }^{38}$.

Por otro lado, todo juicio estético de reflexión, i.e. aquél a cuya base encontramos el sentimiento de placer que ocasiona la conciencia de la mutua conformidad de nuestras facultades representativas en su libre juego, aun cuando no se ve constreñido por ningún concepto en particular, se presenta con la pretensión de universalidad y necesidad que es propia de un juicio objetivo ${ }^{39}$. En este sentido, cabe plantearse: ¿cómo dar razón de la firmeza con que nos empeñamos en convencer a los demás de la belleza o del carácter sublime de un determinado objeto — como si se tratara efectivamente de una propiedad objetiva y fuera en último término posible zanjar el asunto- a pesar de que escudamos nuestra disconformidad con el juicio estético de otros tras el lugar común «sobre gustos no hay nada escrito»? ${ }^{40}$

La exigencia de una «validez común» (Gemeingültigkeit) o «validez universal subjetiva» (subjektive Allgemeingültigkeit) ${ }^{41}$ del sentimiento de placer que está a la

\footnotetext{
${ }^{36}$ Cfr. Ku, \$28 (Ak, V, 261).

${ }^{37}$ Cfr. GUYER, P., op. cit., p. $115 \mathrm{ss}$.

${ }^{38} \mathrm{Cfr} . \mathrm{Ku}$, Allg. Anmerk. zur Expox. Ästh. reflek. Urteilsk. (Ak, V, 271).

${ }^{39} \mathrm{Cfr}$. Ku, $\$ 32(\mathrm{Ak}, \mathrm{V}, 281)$.

${ }^{40} \mathrm{Cfr}$. Ku, $\$ 56$ (Ak, V, 338).

${ }^{41}$ «Aquí debe señalarse primeramente que una universalidad que no se basa en conceptos de objeto (aun cuando meramente empíricos) no es lógica sino estética, i.e. no contiene una cantidad objetiva del juicio sino una subjetiva, para la que utilizo la expresión validez común [Gemeingültigkeit], que designa la validez, no de la relación de una representación con la facultad de conocimiento sino con el sentimiento de placer y displacer para cada sujeto»; $\mathrm{Ku}$, $\$ 8$ ( $\mathrm{Ak}, \mathrm{V}, 214)$.
} 
base de todo juicio estético de reflexión se funda en la idea de un «sentido común» (《sensus communis»), esto es, aquella capacidad de enjuiciamiento que en su reflexión sobre lo dado toma en consideración la disposición de las facultades representativas del resto de seres racionales ${ }^{42}$. Por "sentido» (Sinn) debe entenderse aquí el efecto de la mera reflexión sobre nuestro espíritu, i.e. la conciencia de la concordancia (Stimmung) que existe entre nuestras facultades, que ocasiona el sentimiento de placer coligado a la representación empírica de un objeto $^{43}$ y que presuponemos común al resto de sujetos («sensus communis aestheticus»). En cuanto juicio estético de reflexión, el juicio estético de lo sublime opera bajo la presuposición de una disposición homogénea de las facultades representativas en nosotros, i.e. la comunidad del sentimiento moral en todo ser racional finito ${ }^{44}$.

\section{La exposición simbólica de conceptos morales particulares}

Como hemos señalado en los apartados precedentes, la relación entre el ámbito estético y el moral pasa principalmente por la posibilidad de proporcionar cierto correlato sensible — sin pretensión cognoscitiva alguna- de la idea de la libertad en cuanto fundamento del campo de lo suprasensible. Así, veíamos cómo por medio de las ideas estéticas se da una exposición indirecta de la idea de la libertad, puesto que esta capacidad de la imaginación para situarse allende lo proporcionado en los sentidos es análoga a la facultad que tiene el ser racional finito para determinarse autónomamente.

Cabe señalar, no obstante, que si bien existe cierta similitud entre las ideas de la razón y las ideas estéticas en el hecho de que ambas se sitúan allende lo dado en la experiencia — tanto que estas últimas constituirían el «opuesto equivalente» (Gegenstück) de las primeras ${ }^{45}$, difieren en el modo en que realizan esta operación de tras-paso. Tanto las ideas de la razón como las ideas estéticas comparten ciertamente el punto de partida, mas divergen en el de llegada: mientras que las ideas de la razón apuntan hacia la existencia de un ámbito de objetos de

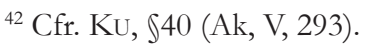

${ }^{43} \mathrm{Cfr}$. Ku, $\$ 40$ (Ak, V, 295).

${ }^{44}$ Cfr. Ku, \$29 (Ak, V, 265).

${ }^{45}$ Cfr. Ku, $\$ 49$ (Ak, V, 313ff).
} 
naturaleza suprasensible, las ideas estéticas emplean el material sensible para proporcionar perspectivas nuevas y enriquecidas de la naturaleza sensible misma ${ }^{46}$.

Las ideas estéticas pueden facilitar cierto correlato intuitivo de ideas de la razón en general y, más específicamente, de conceptos morales particulares. En la exposición simbólica de un concepto de razón, la facultad de juzgar funciona de acuerdo con la mera forma de la reflexión (la acción misma de llevar a unidad lo diverso), siéndole posible asociar una plétora de representaciones que sirven para expresar intuitivamente aspectos de una representación conceptual que carece de un referente sensible directo, i.e. los «atributos estéticos» (ästhetische Attribute $)^{47}$. En este sentido afirma Kant que el poeta sensibiliza ideas — sugiere imágenes mediante un uso elaborado del lenguaje - como puedan ser el reino de los Bienaventurados, el infierno o la eternidad. Así, por ejemplo, la imagen de un águila con un rayo en las garras constituye una representación intuitiva empleada para expresar analógicamente el poderío de una entidad suprasensible como es Júpiter, el rey de los cielos ${ }^{48}$.

En la sensibilización de conceptos de razón particulares, la facultad de juzgar del ser racional finito opera ateniéndose de manera exclusiva a la regla de su proceder reflexivo en general, con el propósito de hallar representaciones que expresen cierto «parentesco» (Verwandtschaft) con el concepto de razón que pretende exponerse, i.e. cierta analogía en los efectos ${ }^{49}$. Es decir: este «mirar de otra manera» aquello que se nos aparece en la intuición con el fin de sensibilizar un elemento que no es susceptible de una exposición directa funciona bajo la úni-

\footnotetext{
${ }^{46}$ Cfr. Makkreel, Rudolf, A., Imagination and Interpretation in Kant, Chicago and London, The University of Chicago Press, 1990, p. 120.

${ }^{47}$ «A aquellas formas que no constituyen la exposición de un concepto dado sino que, en cuanto representaciones concomitantes (Nebenvorstellungen) de la imaginación, expresan tan sólo las consecuencias a él asociadas y el parentesco que tiene con otros, se las denomina atributos (estéticos) de un objeto cuyo concepto, en tanto que idea de la razón, no puede ser expuesto de manera adecuada»; Ku, $\$ 49$ (Ak, V, 315).

${ }^{48}$ Ibíd.

${ }^{49}$ Una de las cuestiones que cabe plantear es hasta qué punto son atribuibles a Kant las objeciones que él mismo realiza al modo de proceder «excesivamente imaginativo» de Herder, que se deja llevar por su espíritu poético en la elaboración de analogías a partir del mundo natural a las que otorga validez cognoscitiva en relación a objetos que escapan a todo conocimiento posible. Es decir: hasta qué punto pretendemos conocer aquello que no entendemos por medio de un elemento que aún conocemos menos; cfr. I Rezension zu J. G. Herders Ideen (Ak, VIII, 53ff).
} 
ca restricción de procurar representaciones que sirvan para presentar intuitivamente la analogía en la relación que se establece entre conceptos de objetos que pertenecen a ámbitos heterogéneos (águila/Júpiter).

Este recurso al mundo sensible para sensibilizar un concepto de razón lo encontramos por ejemplo en la construcción del concepto de derecho, entendido como la posibilidad de una recíproca coacción exterior de los individuos según leyes universales que sea acorde con su libertad de acción, por medio de «la analogía con la posibilidad de un movimiento libre de los cuerpos de acuerdo con la ley de una igualdad de acción-reacción» ${ }^{50}$. Por este motivo, la última definición del gusto proporcionada por Kant es en términos de «la capacidad de enjuiciamiento de la sensibilización de las ideas morales (por medio de cier-

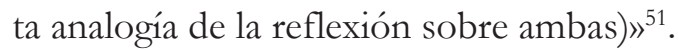

\section{La exposición simbólica de lo bueno en sentido moral}

La facultad de juzgar del ser racional finito presupone la posibilidad de aprehender unitariamente cualquier diversidad dada, concibiendo aquello que se le presenta como si hubiera sido dispuesto por la naturaleza en vistas a las exigencias operacionales de nuestras facultades representativas ${ }^{52}$. Más específicamente, en todo juicio de gusto la facultad de juzgar opera conforme a la idea de una «técnica formal de la naturaleza» (formale Technik der Natur), por medio de la cual concebimos la representación del objeto como si hubiera sido producida con el fin de provocar un sentimiento de placer en nosotros ${ }^{53}$. La facultad de juzgar encierra así una referencia a la idea de un substrato suprasensible de la naturaleza en y fuera de nosotros, en cuanto principio explicativo tanto del armónico funcionamiento de las facultades cognoscitivas del ser racional finito como de la conformidad de la naturaleza con respecto a las mismas ${ }^{54}$.

\footnotetext{
${ }^{50}$ Metaphysik der Sitten (Ak, VI, 232). Puede consultarse al respecto, RENAUT, A., «Introducción» a I. Kant, Métaphysique des Moeurs, Paris, Flammarion, 1994, pp. 33-34.

${ }^{51} \mathrm{Ku}, \$ 60(\mathrm{Ak}, \mathrm{V}, 356)$.

${ }^{52}$ Cfr. I Einleitung KU (Ak, XX, 202).

${ }^{53}$ Cfr. I Einleitung Ku (Ak, XX, 232).

${ }^{54}$ En este sentido afirma Kant explícitamente: «En el gusto, la facultad de juzgar no se ve sometida a una heteronomía de las leyes de la experiencia como en el enjuiciamiento empírico. En vistas de los objetos de un placer tan puro, la facultad de juzgar se proporciona a sí
} 
Es precisamente en la obra de arte que resulta del «genio» (Genie) en el artista donde se patentiza de un modo sensible el fundamento del acorde funcionamiento del conjunto de nuestras facultades. En toda obra de arte bella debe ciertamente advertirse que se trata de una producción humana, sin que pueda no obstante atisbarse el fin a que responde el trabajo del material ${ }^{55}$. Es decir: así como la belleza en la naturaleza presupone la capacidad de enjuiciarla según la analogía con la producción artística, la obra de arte bella debe aparecer como si fuera un producto de la naturaleza misma ${ }^{56}$. La belleza en el arte tan sólo es concebible pues como producto del «genio», i.e. aquel talento o «don natural» (Naturgabe) cuya posesión por parte del artista permite afirmar que es la naturaleza misma la que proporciona la regla al arte ${ }^{57}$. Puesto que en la obra bella no nos es posible hallar una representación bajo la que subsumir de manera plena la forma del objeto, nuestras facultades representativas se demoran en la contemplación del mismo, permitiéndonos apercibir su libre conformidad y, por ende, el substrato suprasensible de la naturaleza en nosotros ${ }^{58}$.

Por otro lado, en relación al substrato suprasensible fuera de nosotros, dado que a la «conformidad a fin» de la naturaleza en el enjuiciamiento de la belleza no puede asociársele ningún fin en concreto (ya que esta conformidad podría explicarse también en términos mecánicos), el ser racional finito tiende a buscarlo en él mismo, hallándolo en aquello que constituye el «fin último» (letater Zweck) de su existencia, a saber, la «determinación moral» (moralische Bestimmung) que le es propia ${ }^{59}$.

misma la ley, así como hace la razón en vistas de la facultad apetitiva. Y, tanto a causa de esta posibilidad interna en el sujeto como de la posibilidad externa de una naturaleza que le es conforme, la facultad de juzgar se ve referida a algo en el sujeto mismo y fuera de él que no es naturaleza, tampoco libertad, pero que está enlazada con el fundamento de la última, a saber, con lo suprasensible, en el que es llevada a unidad la facultad teórica con la práctica de un modo común y desconocido»; Ku, $\$ 59$ (Ak, V, 353). La negrita es nuestra.

${ }^{55}$ En este sentido puede afirmarse que en la obra de arte que es producto del genio en el artista se exhibe la resolución de la tercera antinomia en $\mathrm{KrV}$, en la medida que expone intuitivamente la conjunción entre libertad (entendida en términos de la absoluta originalidad de la obra, i.e. su no sometimiento a normas o reglas) y necesidad (la necesaria sujeción a cierto material en que plasmar esa potencia creativa); cfr. NAHM, M. C., «'Sublimity' and the 'Moral Law' in Kant's philosophy», Kant-Studien 48 (1957), pp. 512-515.

${ }^{56} \mathrm{Cfr}$. KU, $\$ 45$ (Ak, V, 306).

${ }^{57} \mathrm{Cfr}$. KU, $\$ 46$ (Ak, V, 307).

${ }^{58} \mathrm{Cfr}$. Ku, $\$ 57$ (Ak, V, 344).

${ }^{59} \mathrm{Cfr}$. Ku, $\$ 42(\mathrm{Ak}, \mathrm{V}, 301)$. 
De este modo, el sentimiento de placer que está en la base de todo juicio de gusto lleva asociado un interés (intelectual) susceptible de ser emparentado con el ámbito de la moralidad, en la medida en que el «guiño» (Wink) que parece hacernos la naturaleza en la producción de formas bellas es interpretado como el «rastro» (Spur) de una disposición de la naturaleza conforme a los fines constitutivos de la determinación moral en nosotros, algo que nos legitima a esperar la «realización» (Verwirklichung) del Bien supremo en el ejercicio de nuestra liber$\operatorname{tad}^{60}$. Es en este sentido que «lo bello (das Schöne) es el símbolo de lo bueno en sentido moral (das Sittlich-gute) ${ }^{61}$. La facultad de juzgar del ser racional finito contiene pues una referencia a la idea de lo suprasensible en general, en cuanto principio que permite pensar la «conformidad» (Übereinstimmung) entre la naturaleza y los fines de la libertad ${ }^{62}$.

Esta referencia a la idea de un substrato suprasensible no es empero exclusiva del objeto bello. El sentimiento de lo sublime patentiza la inadecuación que media entre nuestra imaginación y la razón en cuanto facultad que exige el absoluto incondicionado en la totalidad de una serie. Esta imposibilidad da no obstante noticia de una dimensión en nosotros que es susceptible de ir más allá de toda magnitud sensible dada y que, por muy confusamente que pueda aparecérsenos, reposa en último término en la determinación suprasensible que es inherente al ser racional finito ${ }^{63}$.

Mediante un determinado empleo de la representación empírica de un objeto, el juicio estético de lo sublime considera lo sensible en la naturaleza como adecuado para un empleo suprasensible del mismo ${ }^{64}$. En la tensión imaginación-

${ }^{60} \mathrm{Cfr} . \mathrm{Ku}, \$ 42(\mathrm{Ak}, \mathrm{V}, 300)$.

${ }^{61} \mathrm{KU}, \$ 59$ (Ak, V, 353). La presente afirmación se halla en el capítulo intitulado «Von der Schönheit als Symbol der Sittlichkeit». Ahora bien: ¿son identificables las expresiones «das Sittlich-gute» $\mathrm{y}$ «Sittlichkeit» por un lado, y «das Schöne» $\mathrm{y}$ «die Schönheit» por otro? ¿Qué está entendiendo propiamente Kant por «das Sittlich-gute»? Es decir: ¿qué es lo que se expone simbólicamente en la belleza/el objeto bello: la idea de la libertad o del Bien supremo? Una discusión en torno al significado preciso de la expresión «das Sittlich-Gute» y su identificación con la idea de Bien supremo entendido en términos de una conducta social fundamentada moralmente (morally grounded social conduct), cfr. FELICITAS MUNZEL, op. cit., \$2. Para una lectura en que se muestra cómo la belleza expone simbólicamente la idea de la libertad, cfr. GUYER, P., op. cit., p. 99.

${ }^{62} \mathrm{Cfr}$. Ku, $\$ 57$ (Ak, V, 346).

${ }^{63} \mathrm{Cfr}$. Ku, $\$ 39$ (Ak, V, 292).

${ }^{64} \mathrm{Cfr}$. Ku, Allg. Anmerk. zur Expos. der Ästh. reflek. Urteil. (Ak, V, 266ff). 
razón, pensamos la naturaleza en su totalidad como el lugar de una posible exposición de las ideas de la razón, una imposibilidad ante la cual lo sublime puede ser descrito en términos de «aquel objeto de la naturaleza, la representación del cual determina al espíritu con vistas a que piense el carácter inalcanzable de la naturaleza en cuanto exposición de ideas ${ }^{65}$. Ahora bien, dado que la pretensión de exhibir lo suprasensible en la naturaleza procede de nosotros mismos, nos vemos obligados a considerar la naturaleza espaciotemporal como mero fenómeno y, por ende, apuntando hacia un substrato suprasensible en cuanto principio de la conformidad entre naturaleza y libertad ${ }^{66}$.

\section{La naturaleza del discurso suprasensible}

Como afirmábamos al inicio del presente trabajo, la reflexión sobre la naturaleza, funcionamiento y validez de nuestro discurso sobre el ámbito de lo suprasensible, en el que debe enmarcarse el moral, cristaliza en los dos modelos que nos permiten pensar toda posible relación de hipotiposis.

Conforme a esta clasificación y a la asunción plena de los rendimientos del juicio teleológico para el conjunto de su pensamiento, Kant presenta en Los avances de la metafísica desde los tiempos de Leibnizy Wolff(1791) una reestructuración del sistema acorde con su concepción de la filosofía como «doctrina de la sabiduría» (Weisheitslehre), i.e. «la ciencia del conocimiento que avanza de lo sensible hacia lo suprasensible por medio de la razón» ${ }^{67}$. En esta obra habla Kant de la posibilidad de una exposición indirecta (o simbólica) de nuestros conceptos de razón, que es aquélla en que se utiliza la representación intuitiva de un objeto para expresar la igualdad en la relación —analogía en los efectos- entre elementos que pertenecen a ámbitos heterogéneos (sensible-inteligible ${ }^{68}$. En este

\footnotetext{
${ }^{65}$ Cfr. Ku, Allg. Anmerk. zur Expos. der Ästh. reflek. Urteil. (Ak, V, 260).

${ }^{66}$ Cfr. Ku, Allg. Anmerk. zur Expos. der Ästh. reflek. Urteil. (Ak, V, 268).

${ }^{67}$ Fortschritte (Ak, XX, 260). Con respecto a la reestructuración del sistema crítico en Los avances de la metafísica desde los tiempos de Leibnizy Wolff [Fortschritte] a partir de la reflexión sobre la naturaleza del juicio teleológico y las modificaciones que supone con respecto a la arquitectónica de 1781, cfr. Turró, S., Tránsito de la naturaleza a la historia en la filosofía de Kant, Barcelona, Ánthropos, 1996, Parte II, Capítulo VI, pp. 207-233.

${ }^{68}$ «A esta acción se la denomina esquematismo cuando la realidad objetiva es asignada al concepto directamente (directe) por medio de la intuición que le corresponde, es decir, cuando este
} 
sentido, la noción de exposición simbólica debe identificarse con el «esquematismo de la analogía» (Schematism der Analogie) que aparece en La religión dentro de los límites de la mera razón (1793) y que se opone al «esquematismo de la determinación del objeto» (Schematism der Objektbestimmung), equivalente al concepto de «exposición esquemática» ${ }^{69}$.

El «esquematismo de la analogía» parte de la constatación de que: a) la razón humana exige hacerse representable incluso aquellos conceptos que escapan por definición a toda sensibilización directa ${ }^{70}$; b) el modo en que esta sensibilización acontece es por medio de una analogía con algo sensible; c) esta exposición no tiene valor constitutivo sino meramente explicativo; es decir, no deben atribuirse al objeto suprasensible aquellas características que resultan del modo en que es en general posible para nosotros un conocimiento del objeto ${ }^{71}$.

Cabe ubicar en este contexto las intrincadas relaciones que establece Kant entre el dominio moral y el ámbito estético. Que la belleza es símbolo de lo bueno en sentido moral significa que en la experiencia de lo bello —en la «conformidad a fin» tanto de nuestras facultades entre sí como del objeto bello con respecto a las mismas - se nos proporciona un referente sensible de la idea de una naturaleza que opera según fines, constituyendo aquella huella que nos permite esperar racionalmente la realización del Bien supremo en el mundo sensible.

concepto es expuesto de manera inmediata. Si el concepto no puede ser expuesto inmediatamente sino tan sólo en sus consecuencias (indirecte), la operación puede ser llamada simbolización del concepto»; Fortschritte (Ak, XX, 279).

${ }^{69}$ «Este es el esquematismo de la analogía (como aclaración), del que no podemos prescindir. Transformar éste en un esquematismo de la determinación del objeto (como ampliación de nuestro conocimiento) es antropomorfismo, el cual es en perspectiva moral (en la religión) de las más perjudiciales consecuencias»; Religion (Ak, VI, 65Anm).

${ }^{70}$ «Es ciertamente una limitación de la razón humana que no puede sin embargo disociársele: que no podemos pensar ningún valor moral de importancia en las acciones de una persona, sin al mismo tiempo hacernos representable de un modo humano la acción o su manifestación. Si bien no quiere afirmarse con ello que sea en ella misma utilizada de ese modo (kat'alétheian); ya que necesitamos siempre de una cierta analogía con los seres naturales para hacernos captable la constitución suprasensible»; Religion (Ak, VI, 64Anm.). La negrita es nuestra.

${ }^{71}$ «Aquí quisiera tan sólo señalar de paso que en el ascenso de lo sensible a lo suprasensible puede uno ciertamente esquematizar (hacer captable un concepto por medio de la analogía con algo sensible); pero de ningún modo puede uno por medio de la analogía con respecto a aquello que corresponde al primero concluir que puede atribuírsele al último también y, de este modo, ampliar su concepto»; Religion (Ak, VI, 65Anm). 
Recogiendo los resultados del análisis del concepto de finalidad en KU, Kant piensa la relación entre el mundo sensible y el mundo inteligible, entre naturaleza y libertad, por analogía con el modelo de producción artesanal: el artesano se sirve de los materiales y del trabajo mecánico sobre los mismos en aras a obtener un producto que responde a los fines a partir de los cuales comenzó su actividad. Por medio de la idea de una «naturaleza artesana» (Natur als Kunst) podemos aunar mecanicismo y finalidad, entendiendo la naturaleza en este sentido en términos de un substrato suprasensible de las dos series ${ }^{72}$.

En este sentido habla Kant en Idea de una historia universal en sentido cosmopolita (1784) de una «intención de la naturaleza» (Naturabsicht) en vistas al pleno desenvolvimiento en la especie de las disposiciones originarias que encontramos en todo ser racional finito ${ }^{73}$. La naturaleza se sirve de la «insociable sociabilidad» (ungesellige Geselligkeit) del hombre para avanzar hacia un ordenamiento legal internacional en que dirimir pacíficamente sus conflictos ${ }^{74}$, puesto que es en una oposición regulada de libertades que el hombre puede desarrollar el conjunto de sus potencialidades ${ }^{75}$. Tiene sentido pues hablar de un «plan oculto de la naturaleza» (verborgener Plan der Natur) en las manifestaciones de la libertad humana (historia) y, por tanto, del mundo como el fruto de la mano de un sabio creador $^{76}$.

Ahora bien, la posibilidad de hermanar ambas perspectivas no excluye el hecho de que haya términos más apropiados para hablar de este substrato supra-

${ }^{72}$ Cfr. Ku, \$75. Al respecto puede consultarse: TuRRÓ, S., Lliçons sobre història i dret a Kant, Barcelona, Edicions de la Universitat de Barcelona, 1993, §3-4. Consúltese también la distinción entre el concepto de físicoteleología y la éticoteleología; $\mathrm{KU}$, §85-86.

${ }^{73}$ Cfr. Idee zu einer allgemeinen Geschichte in weltbürgerlicher Absicht (Ak, VIII, 18). A partir de aquí, citaremos a esta obra con la abreviatura «IdeeG».

${ }^{74}$ Cfr. Zum ewigen Frieden (Ak, VIII, 366ff).

${ }^{75}$ Cfr. IdeeG (Ak, VIII, 28). A este ordenamiento legal internacional lo denomina Kant «liga de pueblos» (Völkerbund) en IdeeG (Ak, VIII, 24), y «federación de estados» (Föderalism) en el «Segundo artículo definitivo para la Paz perpetua»; Zum ewigen Frieden (Ak, VIII, 354ff).

${ }^{76}$ «El hombre quiere concordia; pero la naturaleza sabe mejor aquello que es bueno para su especie: quiere discordia (...). Los móviles naturales para ello, fuente de la insociabilidad y de la oposición general — de los que procede tanto mal pero que provocan una renovada tensión de las fuerzas y, en consecuencia, un mayor desarrollo de las disposiciones naturales - revelan la disposición de un sabio creador y no la mano de un espíritu malvado que hubiera chapuceado o estropeado de un modo envidioso su señorial obra»; IdeeG (Ak, VIII, 21ff); la negrita es nuestra. 
sensible. Una cuestión que cabe plantearse es precisamente cuál es el modo oportuno de referirse a esta naturaleza que despliega un plan en las acciones de los hombres y que, ciertamente, no puede identificarse con la naturaleza causalmecánica de la primera crítica. Es en el opúsculo Hacia la pazperpetua (1795) donde aparece una reflexión metodológica encaminada a establecer el léxico críticamente pertinente — destino, providencia o naturaleza - para denominar a esta entidad racional que actúa teleológicamente ${ }^{77}$.

Considerar el curso de los acontecimientos humanos como orientándose indefectiblemente a la consecución de una federación de estados en que dirimir pacíficamente los conflictos parece invitar al empleo de la expresión «destino» (Schicksal) para referirse al substrato suprasensible de ambas series — sensible e inteligible- Asimismo, porque en la introducción de un hilo conductor en nuestro relato de los acontecimientos históricos consideramos la naturaleza como una entidad racional que interviene subrepticiamente en el curso de la historia, utilízase eventualmente el término «providencia» (Vorsehung) ${ }^{78}$. El término «naturaleza» (Natur) es, sin embargo, el más adecuado para denominar este substrato suprasensible: a) porque se atiene a los efectos del mismo en el mundo sensible según leyes causales; b) y porque corta el paso a peligrosas tentaciones visionarias ${ }^{79}$.

Es precisamente por medio de la actividad artesanal humana y de una concepción técnica de la naturaleza que podemos hacernos representable analógicamente este substrato suprasensible. En el ámbito estético se da pues una exposición simbólica del concepto de lo bueno en sentido moral, en la medida en que el proceder según fines que parece rastrearse en el objeto bello es tomado como una presentación intuitiva de las relaciones que cabe pensar entre la naturaleza en cuanto substrato suprasensible y las dos series fenoménica-inteligible ${ }^{80}$ —bien

\footnotetext{
${ }^{77}$ Cfr. Zum ewigen Frieden (Ak, VIII, 360ff).

${ }^{78}$ Ibíd.

${ }^{79}$ Cfr. Zum ewigen Frieden (Ak, VIII, 362).

${ }^{80}$ Ibíd. En este mismo sentido, la idea de Dios puede entenderse simbólicamente como el campesino (el Demiurgo) que trabaja y da forma a la tierra, introduciendo cierto «orden» en el universo que nos legitima a esperar racionalmente una concordancia última entre el ámbito sensible y el inteligible. Dios es el «sembrador de estrellas» del que habla A. Machado: «Tal vez la mano, en sueños,//del sembrador de estrellas,//hizo sonar la música olvidada// como una nota de la lira inmensa,/ / y la ola humilde a nuestros labios vino//de unas pocas palabras verdaderas»; Machado, A., Soledades. Galerías. Otros Poemas, Madrid, Cátedra, 2000, poema LXXXVIII, p. 211.
} 
entendido que se trata de un determinado punto de vista en la consideración del mundo (Gesichtspunkt der Weltbetrachtung) sin pretensión cognoscitiva alguna- ${ }^{81}$.

Es decir: para poder hablar de la naturaleza como una entidad racional que actúa según fines y, en consecuencia, descubrir una regularidad en el curso de los acontecimientos históricos, el observador debe situarse en el «punto de vista» (Standpunkt) adecuado. De la misma manera que la justificación de la posibilidad del conocimiento exige asumir la función constitutiva que juega el sujeto en la síntesis de lo dado, la posibilidad de pensar una conformidad entre el ámbito sensible y el inteligible requiere situarse desde la perspectiva de la dimensión moral en el hombre ${ }^{82}$ y reconocer la capacidad que tiene para determinarse a obrar de acuerdo con la ley moral, deviniendo productor de un mundo moral ${ }^{83}$.

\section{La educación estética del hombre}

De la repetida contemplación de la belleza resulta una disposición espiritual que, por cuanto refrena las pasiones del alma y el apremio de la inmediatez, es del todo adecuada para una consideración desinteresada de los demás en la toma de decisiones ${ }^{84}$. Es en este contexto que cabe entender la afirmación kantiana por la que se considera que cierta sensibilidad en el espíritu - fruto del talento o del trabajo - para la contemplación de la belleza en la naturaleza es un rasgo indicativo de un alma moralmente buena ${ }^{85}$. Una educación estética favorece pues la capacidad del sujeto para ponerse en el lugar del otro, algo que ciertamente avanza

${ }^{81}$ Cfr. IdeeG (Ak, VIII, 30).

${ }^{82}$ Cfr. Erneuerte Frage: Ob das menschliche Geschlecht im beständigen Fortschreiten zum besseren sei (Ak, VII, 83). A partir de aquí, citaremos a esta obra con la abreviatura «Erneuerte Frage».

${ }^{83}$ En este sentido, afirma Kant: «Pero, ¿cómo es posible una historia a prior? Respuesta: cuando el mismo que adivina produce y organiza los acontecimientos que anuncia de antemano»; Erneuerte Frage (Ak, VII, 79ff). Una presentación de la última sistematización de la filosofía trascendental por la que se concibe al sujeto como productor del «mundo histórico», en claro avance de los subsiguientes desarrollos idealistas, puede encontrarse en TurRó, S., Tránsito de la naturaleza a la historia en la filosofía de Kant, Tercera parte, III.

${ }^{84}$ Cfr. Ku, $\int 59$ (Ak, V, 354).

${ }^{85} \mathrm{Cfr} . \mathrm{Ku}, \mathbb{\int} 42$ (Ak, V, 298ff). 
hacia la moralidad entendida como la capacidad de universalizar nuestras máximas $^{86}$. Por otro lado, una educación en el sentimiento de lo sublime prepara para la «conversión de los corazones», i.e. la adopción de la ley moral como único motivo impulsor de nuestra acción, precisamente porque en él se da la conciencia de una facultad en nosotros que va más allá de las limitaciones del mundo sensible ${ }^{87}$.

En cuanto facultad de las ideas estéticas en el arte ${ }^{88}$, uno de los rasgos característicos del genio es su capacidad para «crear otra naturaleza» a partir de los materiales que le son dados ${ }^{89}$. En el ámbito estético es posible pues ese «mirar de otra manera» por el que nuestra imaginación no se atiene de manera exclusiva a las leyes de asociación que regulan su uso empírico y puede considerar la naturaleza sensible como un «modelo» o «tipo» (Typus) de nuestro actuar moral ${ }^{90}$. Es precisamente por medio del concepto meramente formal de «ley», i.e. la validez universal y necesaria de un enunciado con respecto a una diversidad dada, que nos es posible «esquematizar» la ley moral, considerando la naturaleza sensible como modelo en el enjuiciamiento de la máxima de nuestra acción con respecto al concepto de lo moralmente bueno ${ }^{91}$.

Un espíritu educado en la contemplación estética aguza esa capacidad de «mirar de otra manera» que es condición indispensable para exponer la ley moral median-

${ }^{86} \mathrm{Cfr}$. Ku, $\$ 40$ (Ak, V, 294).

${ }^{87}$ Cfr. Religion (Ak, VI, 50). El carácter desinteresado del placer estético —el objeto bello place en el mero enjuiciamiento de la forma, sin presuponer ningún tipo de interés en la existencia del mismo, y el objeto sublime en su ir contra nuestro interés sensible- donde radica en último término la posibilidad de que el ámbito estético sensibilice el campo de la moralidad, sin que se vea menguada su independencia tanto con respecto al dominio teórico como al práctico; cfr. GUYER, P., op.cit., cap. 3.

${ }^{88} \mathrm{Cfr}$. Ku, $\$ 57$ (Ak, V, 344).

${ }^{89}$ Cfr. MilTon, C. N., «Imagination as the productive faculty for 'Creating another Nature'...», en L. W. Beck (ed.), Proceedings of the Third International Kant Congress, Dordrecht, Reidel Publishing Company, 1972, pp. 442-450.

${ }^{90} \mathrm{Cfr}$. KpV (Ak, V, 68ff). Sobre la «Típica de la facultad de juzgar pura práctica» puede consultarse: ToRRALBA, J. M., «Facultad del juicio y aplicación de la ley moral en la filosofía de Kant», Methodus II (2007), pp. 1-30; del mismo autor, «Kant on the Law of Nature as the Type of Moral Law: On the «Typic of the Faculty of Pure Practical Judgment» and the Good as the Object of Practical Reason», en VVAA, Natural Law: Historical, Systematic and Juridical Approaches, Cambridge Scholars Publishing, 2008, pp. 195-221.

${ }^{91}$ Cfr. Turró, S., «Llei pràctica i esquematització (de Kant a Fichte)», en Anuari de la Societat Catalana de Filosofia, Barcelona, IEC, Número IX, 1997. 
te un uso analógico del concepto de naturaleza sensible ${ }^{92}$. Así, cabe afirmar que es precisamente por medio de la conciencia de nuestra independencia con respecto a la contemplación de la naturaleza desde el prisma de la experiencia ganada en la experiencia estética - donde radica el fundamento último de la posibilidad de todo enjuiciamiento moral, por cuanto permite precisamente esa consideración analógica de la naturaleza por la que ésta deviene «modelo»o «tipo» de lo suprasensible ${ }^{93}$.

\section{Conclusión}

Dos son los modelos que nos permiten pensar toda relación particular-tipo: el concepto de exposición esquemática (o esquematismo de la determinación del objeto) y el de exposición simbólica (o esquematismo de la analogía). En el dominio teórico, la sensibilización de nuestros conceptos empíricos y sensibles puros tiene lugar por medio del esquema en cuanto modo general de proceder por el que la imaginación puede proporcionar una imagen determinada del concepto en cuestión. Por otro lado, parece ser algo específico de las facultades representativas del ser racional finito la exigencia de un correlato sensible, incluso para aquellas representaciones que rehúyen toda posible exposición directa en la intuición. Debe introducirse pues un modelo desde el que pensar la relación particular(sensible)-tipo(suprasensible), salvaguardando la idiosincrasia de estos conceptos y sin que tal relación detente pretensión cognoscitiva alguna. La noción de «exposición simbólica» avanza en esta dirección ${ }^{94}$.

\footnotetext{
92 Acerca de la recepción de la estética kantiana por parte de Schiller, especialmente en lo que tiene que ver con la relación entre la belleza y la educación político-moral del hombre, véase el apartado «Schiller: Aesthetic Education and the Experience of Freedom» en GuYER, P., op. cit., pp. 116-130.

${ }_{93}$ «El arte poético fortalece la mente (Gemüt), en la medida en que le deja sentir su capacidad libre, espontánea e independiente de la determinación de la naturaleza, una capacidad para considerar y enjuiciar la naturaleza en cuanto fenómeno según perspectivas que ella misma no proporciona en la experiencia ni al sentido ni al entendimiento, y utilizarla por tanto para tal fin y, por decirlo así, como esquema de lo suprasensible»; KU, \53 (Ak, V, 326).

${ }^{94}$ La noción de «tipo», en cuanto fundamento de la relación ley moral/máxima de la acción, no coincide plenamente con ninguno de los dos modelos de exposición presentados: a) como consecuencia de su carácter no sensible, el concepto meramente formal de ley no puede considerarse un «símbolo» de la ley moral; b) tampoco puede denominársele propiamente «esquema»,
} 
Que la intuición de un objeto actúa como símbolo de un concepto de razón significa que es posible establecer una analogía en la relación que mantienen dos pares de elementos heterogéneos entre sí. Así, por ejemplo, un molino de viento puede ser utilizado como símbolo de un estado despótico, en la medida en que el funcionamiento automático y uniforme del primero exhibe de manera intuitiva la organización de aquel estado en que los súbditos se conducen con respecto al déspota como las aspas del molino con respecto al eje del mismo.

En el ámbito estético se da una exposición simbólica de distintos aspectos de la dimensión moral del ser racional finito. La «conformidad a fin» sin fin determinado alguno en la representación del objeto bello es análoga a la mera «conformidad a ley» que reviste todo actuar moralmente correcto; en este sentido, la idea de la libertad —en cuanto concepto sobre el que se fundamenta el dominio moral — halla su correlato sensible en la experiencia de la belleza, así como en el objeto sublime en cuanto descubre una dimensión en nosotros que es capaz de situarse allende lo dado en la sensibilidad, elevándose por encima de nuestra animalidad.

Por otro lado, en cuanto representaciones que dan mucho que pensar, las ideas estéticas no sólo revelan la capacidad que tiene la imaginación para no atenerse de manera exclusiva a las leyes de asociación que regulan su uso empírico, sino que le habilitan para proporcionar cierto contenido intuitivo a representaciones que no son susceptibles de una exposición directa en la intuición, como puede ser el caso de la idea de destino, amor o eternidad. Por último, que el objeto bello da la impresión de haber sido dispuesto para acomodarse a nuestras facultades representativas permite considerarlo como símbolo de la idea de lo bueno en sentido moral. De la misma manera que en el objeto bello parece traslucirse una finalidad en el uso de los materiales, cabe concebir el mundo sensible en su totalidad - presentado como mero fenómeno, en su incapacidad para exponer las ideas de la razón, en la experiencia de lo sublime- como habiendo sido producido para la realización de los fines morales en el ser racional finito.

dada su independencia con respecto a la forma a priori originaria de la sensibilidad (el tiempo). Este es el motivo por el que Kant introduce el término «tipo» (Typus), en cuanto designa un modo de exposición que no se ajusta del todo a los dos presentados en el parágrafo 59 de Ku; cfr. MuMBRÚ, A., «Informe de la tesi doctoral: El problema de la sensibilització de l’àmbit de la moralitat a Kant», en Anuari de la Societat Catalana de Filosofia, Barcelona, IEC, número XXI, 2011, pp. 159-162. 
Esta íntima imbricación entre el ámbito moral y el estético justifica el valor moral de una educación estética del hombre. De la repetida contemplación de la belleza, en cuanto refrena los impulsos de la inmediatez, resulta una disposición en el sujeto apropiada para la consideración de los intereses de los demás en la toma de decisiones. Asimismo, ante el carácter inconmensurable o la absoluta potencia de un objeto, el sentimiento de lo sublime descubre una dimensión en el ser racional finito que se caracteriza por ir más allá de lo que proporcionan los sentidos y que se impone incondicionalmente a todo interés sensible. El sentimiento de lo sublime prepara pues para el efecto que el concepto de deber ocasiona en el conjunto de nuestras facultades, dada la clara analogía que media entre el efecto del objeto sublime y el sentimiento moral. Por último, es propio del ámbito estético esa capacidad para «mirar de otra manera» aquello que se nos presenta en que reside el fundamento de la posibilidad de nuestro enjuiciamiento moral, esto es, la consideración de la naturaleza sensible como «tipo» de nuestro actuar moral.

\section{Bibliografía}

\section{Fuentes}

KANT, I. (1902ss.). Kants gesammelte Schriften, Berlin, hrsg. von der Königlichen Preubischen Akademie der Wissenschaft, 23 vol.

\section{Estudios}

BECK, L. W. (1972). Proceedings of the Third International Kant Congress, Dordrecht: Reidel Publishing Company.

BuTTS, R. E. (1969). «Kant's Schemata as Semantical Rules», en L. W. Beck (ed.), Kant's Studies Today, Illinois: Open Court, pp. 290-300.

CASSIRER, E. (1956). El problema del conocimiento en la filosofía y en la ciencia modernas (vol. II), México: Fondo de Cultura Económica.

CoHEn, T. (1982). «Why Beauty is a Symbol of Morality?», en T. Cohen (ed.), Essays in Kant's Aesthetics, Chicago: The University of Chicago Press, pp. 221-236.

Dimitrios, M. (1982). «Das Problem der Sprache bei Kant», en Scheer, B. \& Wohlfart, G., (ed.), Dimensionen der Sprache in der Philosophie des Deutschen Idealismus, Würzburg: Königshausen \& Neumann, pp. 110-154. 
Düssing, K. (1995). «Schema und Einbildungskraft in Kants Kritik der reinen Vernunft», en Lothar Kreimendahl (ed.), Aufklärung und Skepsis. Studien zur Philosophie und Geistesgeschichte des 17. und 18. Jahrbunderts [Festschrift für Günter Gawlick], Stuttgart: Frommann-holzboog, pp. 47-71.

Felicitas Munzel, G. (1995). «»The Beautiful is the Symbol of the Morally-Good». Kant's Philosophical Basis of Proof for the Idea of the Morally-Good», en Journal of the History of Philosophy 33, pp. 301-330.

FLACH, K. (1982). «Zu Kants Lehre von der symbolischen Darstellung», en Kant-Studien 73 , pp. $452-462$.

Grondin, J. (2000). «Zur Phänomenologie des moralischen 'Gesetzes'. Das kontemplative Motiv der Erhebung in Kants praktischer Metaphysik», en Kant-Studien 91, pp. 385-394.

GuYER, P. (1993). Kant and the Experience of Freedom, New York: Cambridge University Press.

HannA, R. (1990). «Kant's Theory of Empirical Judgement and Modern Semantics», en History of Philosophy Quarterly, vol. 7, nº 3, pp. 335-351.

- (1993). «The trouble with Truth in Kant's Theory of Meaning», en History of Philosophy Quarterly, vol. 10, no 1, pp. 1-20.

Heidegger, M. (1991). Kant und das Problem der Metaphysik, 5. Auflage, Frankfurt am Main: Vittorio Klostermann (trad. esp. Kant y el problema de la metafísica (1986), México, FCE).

JanKe, W. (1968/69). «Das Symbol», en Philosophisches Jahrbuch, 76, pp. 164-180.

KaUlBACH, F. (1965). «Schema, Bild und Modell nach den Voraussetzungen des Kantischen Denkens», en Studium Generale 18, pp. 464-479.

LOHMAR, D. (1991). «Kants Schemata als Anwendungsbedingungen von Kategorien auf Anschauungen», en Zeitschrift für philosophische Forschung 45, pp. 77-92.

— (2003). «Husserl's Type and Kant's Schemata. Systematic Reasons for Their Correlation or Identity», en D. WeLTON (ed.), The New Husserl, Indiana University Press, pp. 93-124.

LÜTHE, R. (1984). «Kants Lehre von den ästhetischen Ideen», en Kant-Studien 75, pp. 6574.

Machado, A. (2000). Soledades. Galerías. Otros Poemas, Madrid: Cátedra.

MAKKREEL, Rudolf, A. (1990). Imagination and Interpretation in Kant, Chicago and London: The University of Chicago Press.

Martínez MarzoA, F. (1987). Desconocida raín, común (Estudio sobre la teoría kantiana de lo bello), Madrid: ed. Visor. 
- (1989). Releer a Kant, Barcelona: ed. Anthropos.

Milton, C. N. (1972). «Imagination as the productive faculty for 'Creating another Nature'...», en L. W. Beck (ed.), Proceedings of the Third International Kant Congress, Dordrecht: Reidel Publishing Company, pp. 442-450.

NAHM, M. C. (1957). «'Sublimity' and the 'Moral Law' in Kant's philosophy», en KantStudien 48, pp. 502-24.

Nolan, J. P. (1979). «Kant on Meaning: Two Studies», en Kant-Studien 70, pp. 113-130.

PATON, H. J. (1947). The Categorical Imperative, London: Hutchinson \& Ltd.

Philonenko, A. (1972). L'oeuvre de Kant, 2 vol., París : Librairie Philosophique J. Vrin.

PIPPIN, R. B. (1976). «The Schematism and Empirical Concepts», en Kant-Studien 67, pp. 156-171.

Renaut, A. (1994). «Introducción» a I. Kant, Metaphysique des Moeurs, París : Flammarion.

Rogerson, K. F. (2004). «Kant on Beauty and Morality», en Kant-Studien 95, pp. 338354.

STRAWSON, P. (1975). Los límites del sentido, Madrid: ed. Revista de Occidente.

ThOMPSON, MANLEY (1972). «Singular Terms and Intuitions in Kant's Philosophy», en Review of Metaphysics, pp. 314-43.

TOrRalBA, J. M. (2007). «Facultad del juicio y aplicación de la ley moral en la filosofía de Kant», en Methodus II, pp. 1-30.

- (2008). «Kant on the Law of Nature as the Type of Moral Law: On the «Typic of the Faculty of Pure Practical Judgment» and the Good as the Object of Practical Reason», en VVAA, Natural Law: Historical, Systematic and Juridical Approaches, New York: Cambridge Scholars Publishing, pp. 195-221.

TURRÓ, S. (1993). Lliçons sobre bistòria i dret a Kant, Barcelona: Edicions Universitat de Barcelona.

- (1996). Tránsito de la naturaleza a la historia en la filosofía de Kant, Barcelona: ed. Anthropos.

- (1997). «Llei pràctica i esquematització (de Kant a Fichte)», en Anuari de la Societat Catalana de Filosofia, Número IX, pp. 83-98.

VILLERS, J. (1997). Kant und das Problem der Sprache, Konstanz: Verlag am Hockgraben.

Recibido: $12 / 10 / 2011$

Aceptado: 3/02/2012 